\title{
系列红色染料的合成、光学性质及在蛋白标记中的应用
}

\author{
李慧芳冷 金金间立润杨秉勤* 白银娟* \\ (西北大学化学与材料科学学院 合成与天然功能分子化学教育部重点实验室 西安 710127)
}

\begin{abstract}
摘要 以 3- $N, N$-二乙基氨基酚为原料合成了一系列苂光染料, 用 ${ }^{1} \mathrm{H} N M R,{ }^{13} \mathrm{C} N \mathrm{NR}, \mathrm{IR}, \mathrm{HRMS}$ 对结构进行了表征, 检 测了新型染料的光学性质, 标记了牛血清白蛋白, 比较了新染料与菁染料 $(\mathbf{C y} \mathbf{3})$ 在凝集素微阵列芯片上的荧光信号值. 结果显示, 新型染料的最大发射波长均在 $600 \mathrm{~nm}$ 以上, 斯托克斯位移约 $70 \mathrm{~nm}$ 左右, 染料/蛋白质(D/P)标记效率高达 $1.5 \sim 1.8$, 在凝集素微阵列中新染料和 $\mathbf{C y} 3$ 的荧光信号值相当. 表明新染料可以作为荧光标记物用于凝集素微阵列中检 测糖链的变化.
\end{abstract}

关键词＼cjkstart荧光染料; 合成; 光学性质; 蛋白标记; 凝集素微阵列

\section{A Series of Red Dyes: Synthesis, Optical Properties and Application in Protein Labeling}

\author{
Li, Huifang Leng, Xin Yan, Lirun Yang, Bingqin* Bai, Yinjuan* \\ (Key Laboratory of Synthetic and Natural Functional Molecule Chemistry of Ministry of Education, \\ College of Chemistry \& Materials Science, Northwest University, Xi'an 710127)
}

\begin{abstract}
A series of red fluorescent dyes were synthesized using 3- $N, N$-diethylaminophenol. Their structures were characterized by ${ }^{1} \mathrm{H}$ NMR, ${ }^{13} \mathrm{C}$ NMR, IR and HRMS. The optical properties of these dyes were detected. Bovine serum albumin was labeled by new dyes. The fluorescence signal values of these dyes and cyanine-3 (Cy3) in lectin microarrays were compared. The results show that the maximum emission wavelengths of these dyes are above $600 \mathrm{~nm}$ and the Stokes shifts are about 70 $\mathrm{nm}$. The dye/protein $(\mathrm{D} / \mathrm{P})$ labeling efficiencies of these dyes are as high as $1.5 \sim 1.8$. The fluorescence signal of new dyes is equal to $\mathbf{C y} \mathbf{3}$ in lectin microarray. Hence, these dyes can also be used as fluorescent labels for detection of sugar chain changes in lectin microarrays.

Keywords fluorescent dye; synthesis; spectral property; protein label; lectins microarray
\end{abstract}

蛋白质糖基化是最常见的翻译后修饰(PTM)之一. 它涉及在蛋白质骨架的特定位点上附着碳水化合 物 ${ }^{[1]}$. 因此聚糖在涉及健康和疾病的生理事件中起着 关键的作用 ${ }^{[5 \sim 7]}$. 目前在糖链结构的解析中, 苂光标记 的凝集素微阵列芯片技术成为主要的方法 ${ }^{[8]}$, 该方法可 高通量、系统、灵敏地检测待测样品表面糖链与凝集素 的特异性结合反应, 并且固定凝集素的方式简单、快速 且成本较低. 在使用的各种荧光标记染料中, 近红外染 料 $(>600 \mathrm{~nm})$ 的应用研究引起了人们较大的兴趣, 这类 染料对生物样品光损伤小, 组织穿透深度大, 且生物分 子的背景苂光干扰小 ${ }^{[9 \sim 11]}$, 在苂光标记的凝集素微阵列 芯片技术的应用中, 目前使用较多的是菁染料系

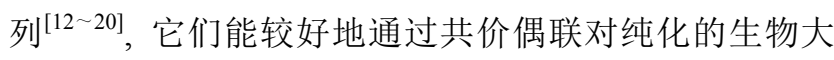
分子进行标记 ${ }^{[21]}$. 但这类染料也因结构的差异存不同 的缺点. 有些染料斯托克斯位移较小, 应用过程中常常 导致自猝灭现象的发生，造成苂光检测中的误差，有些 染料光学性能虽好, 但合成过程复杂, 价格昂贵, 限制 或降低了使用范围. 如何开发出光学性能良好且价格低 廉的新型荧光染料用于标记蛋白, 是一项具有现实意义 的研究课题.

我们课题组在查阅大量文献资料的基础上，设计合 成了一系列氧杂多环结构荧光染料 HQ1 HQ4. 首先 以 $3-N, N$-二乙基氨基酚为原料以 3 步反应且较高收率得 到系列新型红色荧光染料 $\mathrm{HQ1} \sim \mathrm{HQ} 4$, 发射波长均大

* Corresponding authors. E-mail: yangbq@nwu.edu.cn; baiyinjuan@nwu.edu.cn Received July 22, 2018; revised September 11, 2018; accepted October 26, 2018. Project supported by the National Natural Science Foundation of China (No. 21172178). 国家自然科学基金(No. 21172178)资助项目. 
于 $600 \mathrm{~nm}$. 用 ${ }^{1} \mathrm{H}$ NMR, ${ }^{13} \mathrm{C}$ NMR, IR, HRMS 对结构进 行了表征, 检测了新型染料的光学性质, 斯托克斯位移 增加到 $70 \mathrm{~nm}$ 以上, 具有良好的光稳定性和水溶性. 对 牛血清白蛋白(BSA)进行标记纯化后测得这些染料的标 记蛋白的染 料/蛋白质 $(\mathrm{D} / \mathrm{P})$ 值高达 $1.5 \sim 1$.9. 而且凝集 素芯片扫描结果显示, 新染料的荧光信号值与 $\mathbf{C y 3}$ 相 当. 表明新型染料在有关蛋白标记的研究中具有较好实 用价值.

\section{1 结果与讨论}

\section{1 染料 $\mathrm{HQ} 1 \sim \mathrm{HQ} 4$ 的合成}

标记蛋白的染料分子, 结构中必须具有能够插入蛋 白链中的烷基链羧基或氨基, 设计合成了带有支链羧基 的目标化合物 HQ1 $\sim$ HQ4, 合成路线如 Scheme 1 所示. 将 3- $N, N$-二乙基氨基酚和氢氧化钠溶于乙腈中, 反应 3 $\mathrm{h}$ 后加入对甲苯磺酸甲酯反应 $6 \mathrm{~h}$, 得到中间体 $\mathbf{a}$, 产率 95\%. 用中间体 $\mathbf{a}$ 和琥珀酸酐通过傅克酰基化反应合成 中间体 b. 中间体 b 在强酸甲烷磺酸中与取代的狮酮 d1 d4 反应生成目标化合物 HQ1 HQ4, 产率约 70\%.

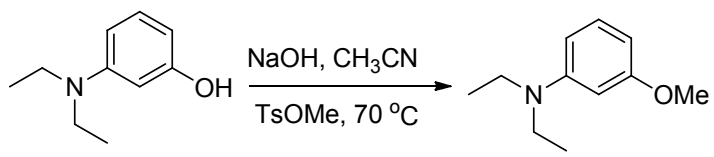

a<smiles>CCN(CC)c1ccc(C(=O)CCC(=O)O)c(O)c1</smiles>

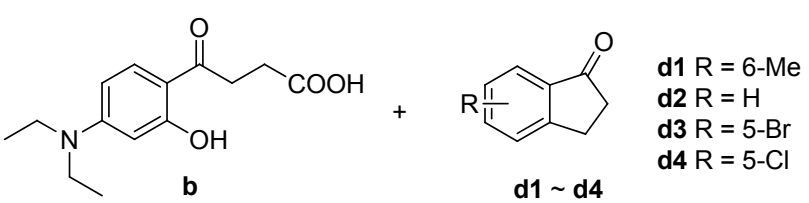<smiles>[R]c1cc2c3oc4cc(N(CC)CC)ccc4c(CCC(=O)O)c(Cc4ccc(O)cc4)c=3c2cc1O</smiles>

图式 1 荧光染料 HQ1 HQ4 的合成

Scheme 1 Synthesis of fluorescent dyes HQ1 HQ4

\section{2 染料 $\mathrm{HQ} 1 \sim \mathrm{HQ} 4$ 的光学性能}

染料 HQ1 HQ4 在不同溶剂中的吸收光谱和发射 光谱如图 1 和 2 . 光谱图中所用的溶液浓度为 $1 \times 10^{-5}$ $\mathrm{mol} \cdot \mathrm{L}^{-1}$, 相关数据在表 1 中. 从表 1 看出这些染料在不 同溶剂中发射波长均在 $600 \mathrm{~nm}$ 以上，而且斯托克斯位 移在 $70 \mathrm{~nm}$ 以上. 染料中不同取代基团 $\mathrm{CH}_{3}, \mathrm{H}, \mathrm{Cl}, \mathrm{Br}$ 在不同溶剂中，荧光发射波长相差不大. 同时发现不同 溶剂对这些化合物吸收波长影响不大，但对吸光度有明 显影响(图 1,2). 在二甲基亚砜(DMSO)中可见光区没有 明显的吸收峰, 在水、甲醇和 DMSO 中的荧光强度较弱, 在二氯甲烷中染料 HQ1 HQ4 系列苂光强度较强. 这 些现象可能是溶剂的极性所致, 染料分子与极性溶剂之 间发生了不同程度的配位作用, DMSO 配位作用较大导 致吸光度最小.

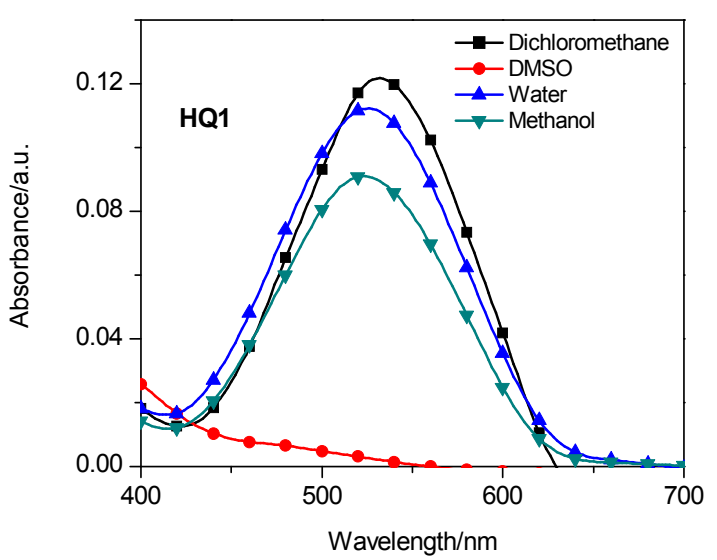

图 1 HQ1 在各种溶剂中的吸收光谱

Figure 1 Absorption spectra of HQ1 in different solvents

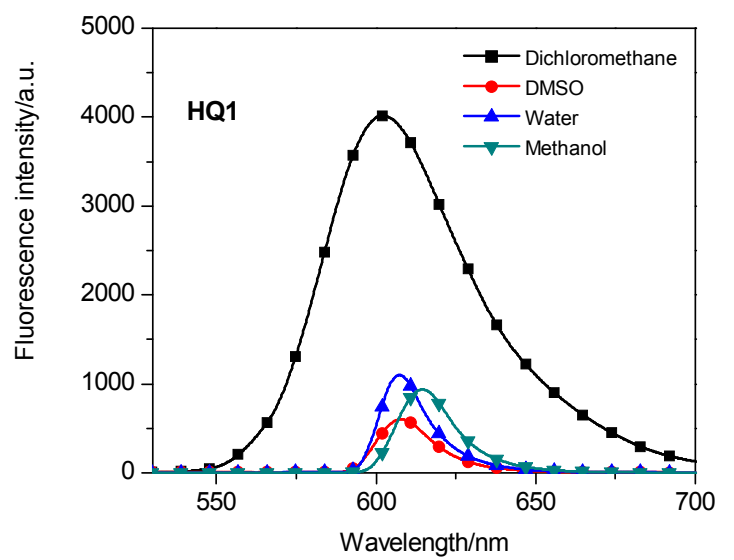

图 $2 \mathrm{HQ1}$ 在各种溶剂中的发射光谱(激发波长: $535 \mathrm{~nm}$ ) Figur 2 Emission spectra of HQ1 in different solvents $\left(\lambda_{\mathrm{ex}}: 535\right.$ $\mathrm{nm})$

优良的光稳定性是近红外染料的重要指标之一 ${ }^{[22]}$. 采用连续照射法进行了光稳定性测试(图 3). 在磷酸缓 冲液(PBS)中, 将染料 HQ1 HQ4 放置在 $500 \mathrm{~W}$ 碘铇灯 下连续照射，以紫外吸收强度为输出信号，每隔 $10 \mathrm{~min}$ 检测一次, $60 \mathrm{~min}$ 后, HQ1 $\sim \mathrm{HQ4}$ 的紫外吸收强度随 
表 1 染料 HQ1 HQ4 在不同溶剂中的光谱数据

Table 1 Spectral data of the dyes HQ1 $\sim$ HQ4 in different solvents

\begin{tabular}{|c|c|c|c|c|c|c|}
\hline Dye & Solvent & $\lambda_{\mathrm{abs}} / \mathrm{nm}$ & $\lambda_{\mathrm{em}} / \mathrm{nm}$ & Stokes shift/nm & 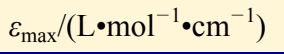 & $\Phi_{F}^{a} / \%$ \\
\hline \multirow{4}{*}{ HQ1 } & $\mathrm{CH}_{3} \mathrm{OH}$ & 532 & 607 & 75 & 15200 & 17 \\
\hline & $\mathrm{CH}_{2} \mathrm{Cl}_{2}$ & 534 & 602 & 68 & 15600 & 35.6 \\
\hline & $\mathrm{H}_{2} \mathrm{O}$ & 535 & 609 & 74 & 17600 & 3.4 \\
\hline & DMSO & - & 605 & - & - & - \\
\hline \multirow{4}{*}{ HQ2 } & $\mathrm{CH}_{3} \mathrm{OH}$ & 534 & 609 & 75 & 21600 & 27 \\
\hline & $\mathrm{CH}_{2} \mathrm{Cl}_{2}$ & 537 & 604 & 67 & 13200 & 71.2 \\
\hline & $\mathrm{H}_{2} \mathrm{O}$ & 531 & 609 & 78 & 19800 & 3.3 \\
\hline & DMSO & - & 604 & - & - & - \\
\hline \multirow{4}{*}{ HQ3 } & $\mathrm{CH}_{3} \mathrm{OH}$ & 532 & 610 & 78 & 5800 & 12 \\
\hline & $\mathrm{CH}_{2} \mathrm{Cl}_{2}$ & 535 & 610 & 75 & 7300 & 23.4 \\
\hline & $\mathrm{H}_{2} \mathrm{O}$ & 530 & 614 & 84 & 14000 & 2.8 \\
\hline & DMSO & - & 607 & - & - & - \\
\hline \multirow{4}{*}{ HQ4 } & $\mathrm{CH}_{3} \mathrm{OH}$ & 536 & 604 & 68 & 10000 & 15 \\
\hline & $\mathrm{CH}_{2} \mathrm{Cl}_{2}$ & 535 & 610 & 75 & 14300 & 64.5 \\
\hline & $\mathrm{H}_{2} \mathrm{O}$ & 532 & 607 & 75 & 10000 & 2.4 \\
\hline & DMSO & - & 610 & - & - & - \\
\hline
\end{tabular}

${ }^{a} \phi_{\mathrm{F}}$ is the relative fluorescence quantum yield estimated by using Rhodamine B as a fluorescence standard.

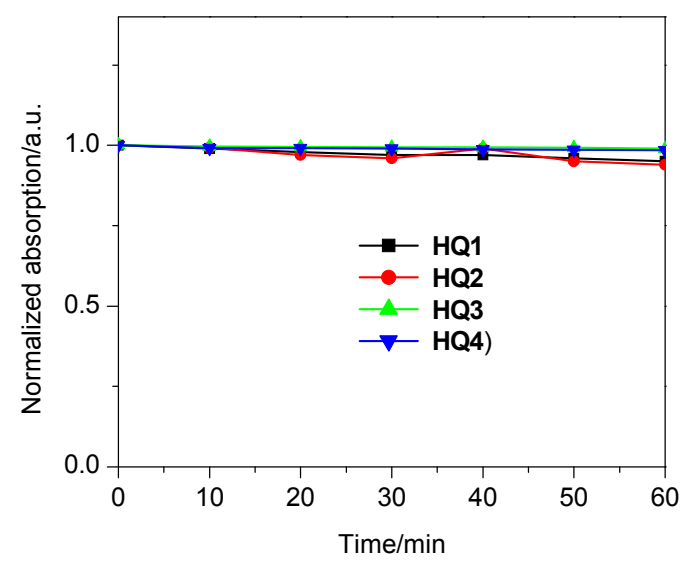

图 3 化合物 HQ1 HQ4 在 PBS 中的光稳性

Figur 3 Photostability of the compounds HQ1 $\sim$ HQ4 in PBS

着时间的延长始终在 $96 \%$ 以上, 表明这些红色染料具有 良好的光稳定性.

在生物学研究中, 染料对于酸碱度的敏感程度是需 考虑的一大因素. 目标染料在 $\mathrm{pH} 3.12 \sim 11.13$ 的范围中, 苂光强度变换的归一化的曲线如图 4 所示. 染料 HQ1 HQ4 在 pH 6.17 9.21 范围内具有很强的苂光, 此范围 正是处于生命体或生物大分子适宜的 $\mathrm{pH}$ 范围, 说明染 料 HQ1 HQ4 在生物学应用中有着较好的适应条件.

染料在水中的溶解度也是衡量染料性能的重要指 标. 图 5 显示了 $\mathrm{HQ1} \sim \mathrm{HQ4}$ 在 PBS 中的工作曲线, 表 2 列出了相应的溶解度数据. 可以看出染料 $\mathrm{HQ1} \sim \mathrm{HQ4}$ 溶解度在 $(3.23 .2 \pm 0.18) \times 10^{-2}$ 和 $(6.96 .9 \pm 0.22) \times 10^{-2}$ $\mathrm{mg} / \mathrm{mL}$ 之间. 其中 HQ3 的溶解性最好.

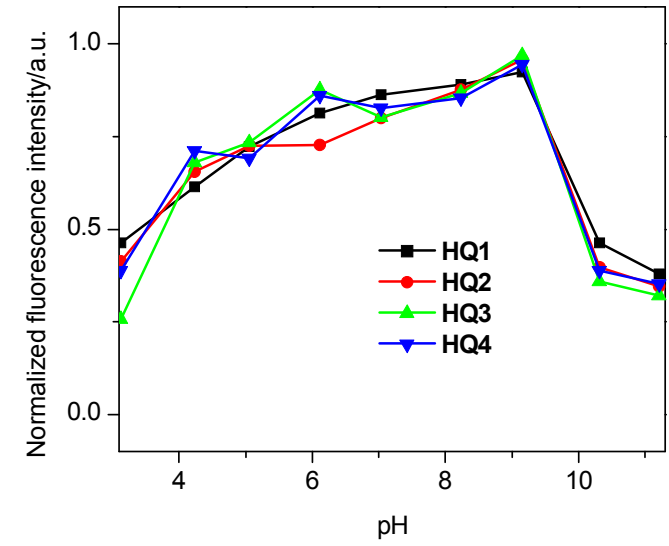

图 $4 \mathrm{HQ1} \sim \mathrm{HQ4}$ 在不同 $\mathrm{pH}$ 下的荧光特性 Figur 4 Fluorescence characteristics of HQ1 $\sim$ HQ4 under different $\mathrm{pH}$

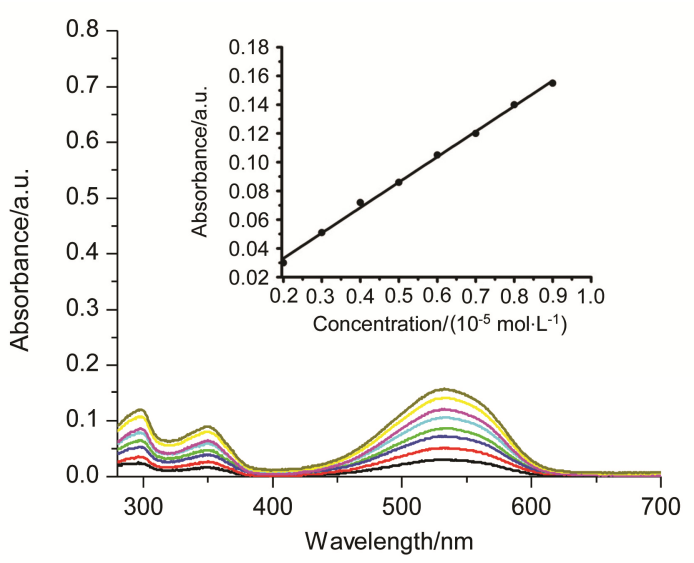

图 $5 \mathrm{HQ4}$ 在 PBS 溶液中的工作曲线

Figur 5 Working curve of HQ4 in PBS solution 
表 2 HQ1 HQ4 在 PBS 溶液中的溶解度

Table 2 Solubility of HQ1 $\sim$ HQ4 in PBS solution

\begin{tabular}{ccc}
\hline Dye & Absorbance/a.u. & Maximum solubility $/\left(10^{-2} \mathrm{mg} \cdot \mathrm{mL}^{-1}\right)$ \\
\hline HQ1 & $2.5 \pm 0.10$ & $3.2 \pm 0.18$ \\
HQ2 & $2.9 \pm 0.10$ & $5.3 \pm 0.16$ \\
HQ3 & $5.5 \pm 0.10$ & $8.3 \pm 0.15$ \\
HQ4 & $3.1 \pm 0.10$ & $6.9 \pm 0.22$ \\
\hline
\end{tabular}

\section{3 染料-BSA 共轭物}

\subsection{1 染料-BSA 共轭物与游离染料的光学性质对比}

图 6 和 7 分别是染料-BSA 的紫外吸收和苂光发射 图, 相应的光谱数据归纳在表 3 中. 由表 3 可知, 这些染 料-BSA 共轭物的紫外-可见吸收和苂光光谱分别在 500 520 和 540 570 nm 之间, 而游离染料的紫外-可 见吸收和苂光光谱分别在 524 和 $600 \mathrm{~nm}$ 以上. 与游离染 料相比, 染料-BSA 共轭物发生蓝移. 比如 HQ1 共价结 合前紫外吸收在 $524 \mathrm{~nm}$ 荧光发射在 $608 \mathrm{~nm}$, 共价结合 后, 蓝移至 498/553.4 nm. 值得注意的是所有染料在共 价结合后都有明显的荧光增强. 例如 HQ1-BSA 在 PBS

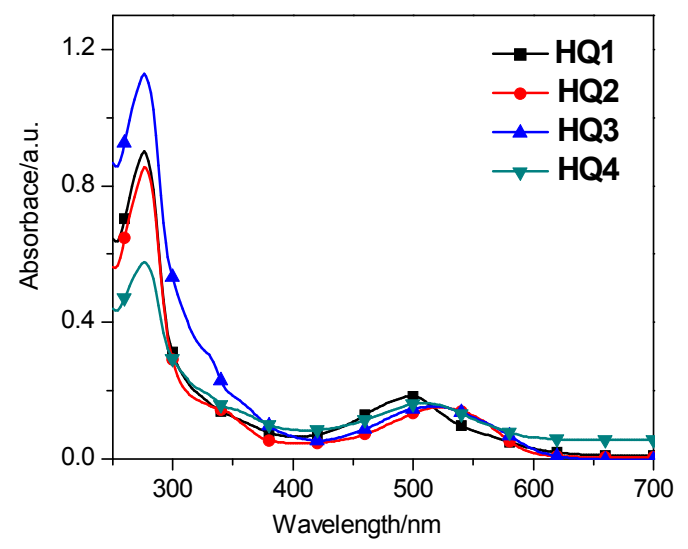

图 6 染料-BSA 在 PBS 中的吸收光谱和发射光谱

Figur 6 Absorption a spectra of dye-BSA in PBS

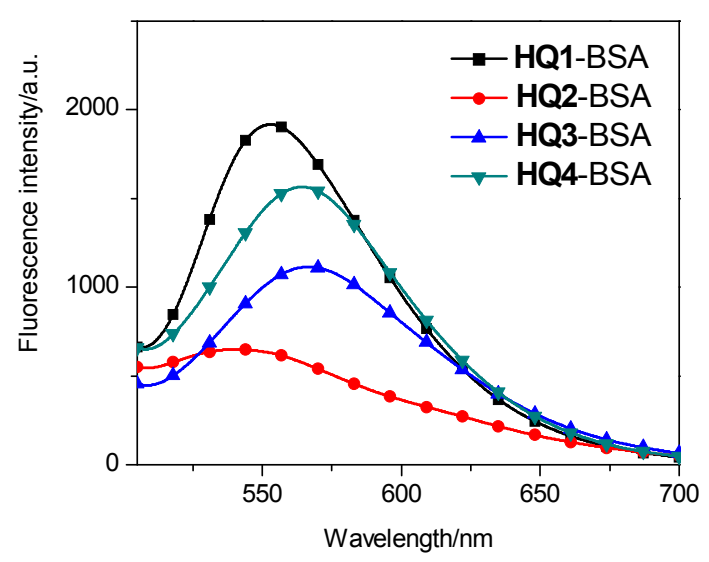

图 7 染料-BSA 在 PBS 中的发射光谱(激发波长: $495 \mathrm{~nm}$ ) Figur 7 Emission spectra of dye-BSA in PBS $\left(\lambda_{\text {ex }}: 495 \mathrm{~nm}\right)$
中的苂光量子产率达到 $6.7 \%$ 左右, 是游离染料的 2 倍. 这种苂光增强效应是由染料结合蛋白质后形成刚性微 环境导致 ${ }^{[23]}$ ，对提高苂光标记蛋白质的检测灵敏度非 常有意义.

表 3 PBS 中游离染料 HQ1 $\sim$ HQ4 和染料-BSA 的光谱数据 Table 3 Spectral data of free dyes HQ1 $\sim$ HQ4 and dye-BSA in PBS

\begin{tabular}{lcccc}
\hline \multicolumn{1}{c}{ Dye } & $\lambda_{\text {abs }} / \lambda_{\text {em }} / \mathrm{nm}$ & Stokes shift $/ \mathrm{nm}$ & $\Phi_{\mathrm{F}}{ }^{a} / \%$ & $\Phi_{\text {Fdye-BSA }} / \Phi_{\text {Fdye }}$ \\
\hline HQ1 & $528 / 608$ & 80 & 3.5 & \\
HQ2 & $524 / 608$ & 84 & 2.8 & \\
HQ3 & $524 / 614$ & 90 & 5.1 & \\
HQ4 & $530 / 605$ & 75 & 2.5 & \\
HQ1-BSA & $498 / 553$ & 55 & 6.7 & 1.9 \\
HQ2-BSA & $522 / 541$ & 19 & 3.4 & 1.2 \\
HQ3-BSA & $516 / 567$ & 51 & 5.7 & 1.1 \\
HQ4-BSA & $526 / 565$ & 39 & 3.2 & 1.3 \\
\hline
\end{tabular}

\subsection{2 染料的 $\mathrm{D} / \mathrm{P}$ 值的计算}

在相同的测试条件下, 以 BSA 为模型蛋白, 用目标 染料对其进行标记, 标记纯化后计算这些染料的标记效 率如表 4 所示, 这些染料的 $\mathrm{D} / \mathrm{P}$ 在 $1.5 \sim 1.9$. 在生物标记 中标记蛋白质最适合的 $\mathrm{D} / \mathrm{P}$ 为 $1.0 \sim 2.0^{[24]}$. 因此新染料 HQ1 HQ4 对蛋白质有较高的标记效率. 随着取代基 才 $\mathrm{CH}_{3}, \mathrm{H}, \mathrm{Cl}, \mathrm{Br}$ 的变化, $\mathbf{H Q}$ 系列荧光染料的蛋白标记 效率表现为 $\mathrm{HQ1} \approx \mathrm{HQ4}<\mathrm{HQ2}<\mathrm{HQ3}$, 说明取代基为 甲基和氯原子的标记效果稍逊于溴原子和氢原子.

表 4 染料 HQ1 $\sim$ HQ4 对于 BSA 的 D/P 值 Table 4 D/P of HQ1 $\sim$ HQ4 dye for BSA

\begin{tabular}{ccccc}
\hline Dye & HQ1 & HQ2 & HQ3 & HQ4 \\
\hline D/P & 1.53 & 1.74 & 1.97 & 1.52 \\
\hline
\end{tabular}

\section{4 凝集素微阵列中新染料 $\mathrm{HQ} 1 \sim \mathrm{HQ} 4$ 与 Cy3 荧光信 号值的比较}

新染料 HQ1 HQ4 成功地将血清和唾液两种糖蛋 白进行了标记, 之后与 $\mathbf{C y 3}$ 的标记效果进行了对比(图 8), 并与凝集素芯片成功地进行了杂交. 以染料 HQ1 为 例, 杂交斑点形态为圆形, 没有遗漏点及连点, 说明芯 片制作很成功. 此外从斑点杂交看出, 染料 HQ1 与糖蛋 白的结合不影响糖蛋白与凝集素的结合. 用 GenePix 3.0 软件计算了每种糖聚糖结构的凝集素苂光信号值的 百分比. 如图 9 所示, 在血清中凝集素 ECA, HHL, GSL-II, PHA-E, SJA, PNA, EEL, LTL, LEL, PSA, VVA 和 SBA 苂光强度均低于 $\mathbf{C y 3}$. 但凝集素 AAL, WFA, PTL-I, GSL-I, RCA120, STL, PTL-II, DSA, NPA, ACA, UEA-I, PWM 和 SNA 苂光强度均显著高于 Cy3. 其它凝 集素与 $\mathrm{Cy3}$ 基本持平. 在唾液中凝集素 GSL-II, SJA, LTL, MPL, LEL, DBA, ConA, SBA, VVA, PSA, ACA, 
SNA, BPL, MAL, PWM 苂光强度均低于 $\mathbf{C y 3}$, 而凝集素 Jacalin, ECA, HHL, WFA, MAL-II, PHA-E, PTL-I, AAL, RCA120, STL, BSI、PTL-II, UEA-I 和 PHA-E +L 苂光 强度均显著高于 $\mathrm{Cy3}$. 其它凝集素与 $\mathrm{Cy3}$ 基本持平. 这 些结果表明, 新染料可以作为糖蛋白的标记物用于凝集 素微阵列中分析有关糖链的变化.
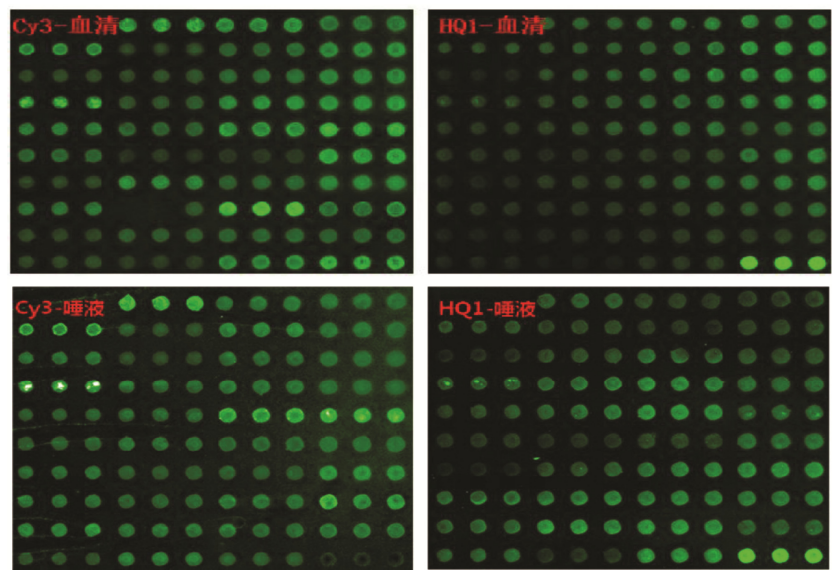

图 8 环氧幻灯片上 Cy3-糖基化和新染料-糖基化后与凝集素 阵列的结合图

Figur 8 Cy3-glycosylation and new dye-glycosylation binding to lectin array on epoxide slides

\section{2 结论}

设计合成了一系列氧杂多环结构染料 HQ1 HQ4,
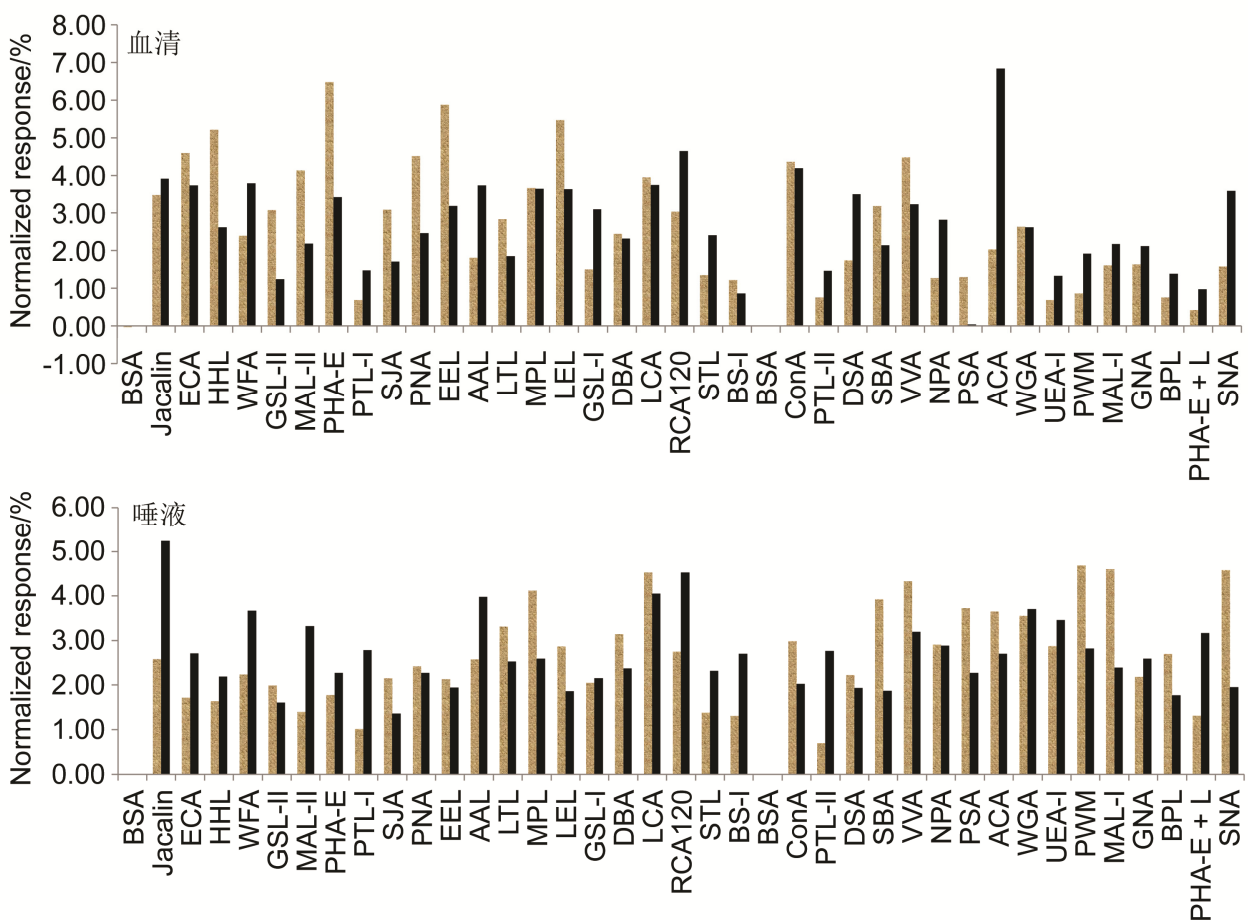

图 9 用 Genepix5.1 软件对图 8 的幻灯片进行分析

Figur 9 Graphical representation of the array data from the slide shown in Figure 8 Slides were analyzed by using the Genepix 5.1 software. The average of the median fluorescence signal (arbitrary units) for a single slide is taken. Standard deviations were calculated by using Microsoft Excel. Solid line: Cy3, Dotted line: HQ1 
磺酸甲酯并升温至 $40{ }^{\circ} \mathrm{C}$, 再反应 $4 \sim 6 \mathrm{~h}$. 反应结束后 过滤得到粗产物, 用石油醚/乙酸乙酯 $(V: V=10: 1)$ 过 柱分离, 得到中间体 a, 产率 $95 \%$.

将 $1.4 \mathrm{~g}$ (8 mmol)中间体 $\mathbf{a}, 1.2 \mathrm{~g}(12 \mathrm{mmol})$ 丁二酸酐 与 $3.7 \mathrm{~g}(28 \mathrm{mmol})$ 无水氯化铝溶于 $100 \mathrm{~mL} \mathrm{CH} \mathrm{Cl}_{2}$ 中, 室温搅拌 $8 \sim 10 \mathrm{~h}$, 之后加入 $50 \mathrm{~mL} 1 \mathrm{~mol} \cdot \mathrm{L}^{-1} \mathrm{HCl}$ 水溶 液, 搅拌成为黄色浑液体, 用 $\mathrm{CH}_{2} \mathrm{Cl}_{2}$ /饱和食盐水 $(V$ : $V=1: 1)$ 萃取, 再无水硫酸钠充分干燥后旋去溶液, 用 乙酸乙酯/石油醚 $(V: V=1: 1)$ 过柱分离, 得中间体 $\mathbf{b}$, 产率 $30 \%$.

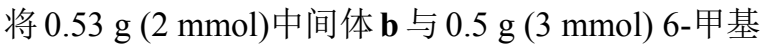
狮酮溶于甲磺酸 $(8 \mathrm{~mL})$ 中, 在 $90{ }^{\circ} \mathrm{C}$ 下搅拌 $12 \mathrm{~h}$, 自然冷 却后加入 $15 \mathrm{~g}$ 冰水混合物, 再用 $\mathrm{CH}_{2} \mathrm{Cl}_{2}$ / 饱和食盐水 $(V: V=1: 1)$ 萃取得到粗产物. 粗品经二氯甲烷/甲醇 $(V: V=10: 1)$ 过柱分离得到目标化合物 HQ1, 产率 70\%. 化合物 HQ3, HQ4 的合成方法类似 HQ1.

10-(2-羧乙基)-7-(二乙基氨基)-3-甲基-11H-狮并 [1,2-b]苯并吡喃-5-鎓 (HQ1): 红色固体 $(0.37 \mathrm{~g})$, 产率 50\%. m.p. 101 102 ${ }^{\circ} \mathrm{C} ;{ }^{1} \mathrm{H}$ NMR (400 MHz, $\left.\mathrm{CDCl}_{3}\right) \delta$ : $8.42(\mathrm{~d}, J=9.6 \mathrm{~Hz}, 1 \mathrm{H}), 7.87(\mathrm{~s}, 1 \mathrm{H}), 7.67$ (d, $J=7.8 \mathrm{~Hz}$, $1 \mathrm{H}), 7.58 \sim 7.49(\mathrm{~m}, 1 \mathrm{H}), 7.30(\mathrm{dd}, J=9.7,2.1 \mathrm{~Hz}, 1 \mathrm{H})$, $6.95(\mathrm{~d}, J=2.2 \mathrm{~Hz}, 1 \mathrm{H}), 4.24(\mathrm{~s}, 2 \mathrm{H}), 3.75 \sim 3.54(\mathrm{~m}, 4 \mathrm{H})$, $3.25(\mathrm{t}, J=7.4 \mathrm{~Hz}, 2 \mathrm{H}), 2.53(\mathrm{~s}, 3 \mathrm{H}), 1.38(\mathrm{t}, J=7.1 \mathrm{~Hz}$, $6 \mathrm{H}) ;{ }^{13} \mathrm{C}$ NMR (101 MHz, DMSO- $\left.d_{6}\right) \delta: 173.2,167.9$, $161.2,158.7,155.1,145.3,138.9,135.5,133.0,129.4$, $128.2,126.5,122.6,117.5,116.2,97.0,45.8,33.9,32.5$, 25.6, 21.4, 12.9; IR (KBr) v: 3419, 2923, 2852, 1635, 1546, 1511, 1346, 1203, 1074, $788 \mathrm{~cm}^{-1}$; HRMS (ESI) calcd for $\mathrm{C}_{24} \mathrm{H}_{26} \mathrm{NO}_{3} 376.1907$, found 376.1920.

10-(2-羧乙基)-7-(二乙基氨基)- $11 H$-狮并 $[1,2-b]$ 吡 喃-5-鎓(HQ2): 红色固体 $(0.31 \mathrm{~g})$, 产率 48\%. m.p. 150 $154{ }^{\circ} \mathrm{C} ;{ }^{1} \mathrm{H}$ NMR (400 MHz, MeOD) $\delta: 8.15$ (d, $J=9.6$ $\mathrm{Hz}, 1 \mathrm{H}), 8.04$ (d, $J=7.7 \mathrm{~Hz}, 1 \mathrm{H}), 7.74$ (d, $J=7.7 \mathrm{~Hz}, 1 \mathrm{H})$, 7.67 (t, $J=7.5 \mathrm{~Hz}, 1 \mathrm{H}), 7.56(\mathrm{t}, J=7.5 \mathrm{~Hz}, 1 \mathrm{H}), 7.35$ (dd, $J=9.6,2.4 \mathrm{~Hz}, 1 \mathrm{H}), 7.18$ (d, $J=2.4 \mathrm{~Hz}, 1 \mathrm{H}), 3.64$ (q, $J=$ $7.1 \mathrm{~Hz}, 4 \mathrm{H}), 3.21(\mathrm{dt}, J=3.2,1.6 \mathrm{~Hz}, 4 \mathrm{H}), 2.71(\mathrm{~s}, 2 \mathrm{H})$, 1.25 (t, $J=7.1 \mathrm{~Hz}, 6 \mathrm{H}) ;{ }^{13} \mathrm{C}$ NMR (151 MHz, DMSO- $d_{6}$ ) $\delta: 173.9,169.1,162.1,159.7,156.1,148.5,134.9,133.8$, $129.9,129.7,128.5,127.3,123.4,118.2,117.1,97.6,46.5$, $34.2,30.2,27.7,13.0$; IR (KBr) v: 3419, 2925, 2856, 1725, 1635, 1513, 1346, 1267, 1083, $725 \mathrm{~cm}^{-1}$; HRMS (ESI) calcd for $\mathrm{C}_{23} \mathrm{H}_{24} \mathrm{NO}_{3} 362.1751$, found 362.1768.

2- 溴-10-(2-羧乙基)-7-(二乙基氨基)-11H-狮并 [1,2-b]吡喃-5-鎓(HQ3): 红色固体(0.28 g), 产率 52\%. m.p. $163 \sim 164{ }^{\circ} \mathrm{C} ;{ }^{1} \mathrm{H}$ NMR (400 MHz, MeOD) $\delta: 8.18$ $(\mathrm{d}, J=9.6 \mathrm{~Hz}, 1 \mathrm{H}), 8.00 \sim 7.82(\mathrm{~m}, 2 \mathrm{H}), 7.70(\mathrm{~d}, J=8.3$ $\mathrm{Hz}, 1 \mathrm{H}), 7.42$ (dd, $J=9.6,2.2 \mathrm{~Hz}, 1 \mathrm{H}), 7.20$ (d, $J=2.2 \mathrm{~Hz}$, $1 \mathrm{H}), 3.74$ (q, $J=7.1 \mathrm{~Hz}, 4 \mathrm{H}), 3.33$ (s, 4H), 2.82 (s, 2H), $1.37(\mathrm{t}, J=7.1 \mathrm{~Hz}, 6 \mathrm{H}) ;{ }^{13} \mathrm{C}$ NMR (101 MHz, DMSO- $\left.d_{6}\right)$ $\delta: 173.0,166.7,160.9,158.8,155.4,148.9,132.0,129.7$, $129.5,129.1,128.2,127.3,123.6,117.6,116.6,97.0,45.7$, 33.2, 31.7, 29.3, 12.1; IR (KBr) v: 3415, 2923, 1758, 2854, 1633, 1511, 1346, 1116, 1039, $840 \mathrm{~cm}^{-1}$; HRMS (ESI) calcd for $\mathrm{C}_{23} \mathrm{H}_{23} \mathrm{BrNO}_{3} 440.0856$, found 440.0857 .

10-(2-羧乙基)-2-氯-7-(二乙基氨基)-11H-狮并 [1,2-b]吡喃-5-鎓(HQ4): 红色固体(0.36 g), 产率 $43 \%$. m.p. $209 \sim 210{ }^{\circ} \mathrm{C} ;{ }^{1} \mathrm{H}$ NMR $\left(400 \mathrm{MHz}, \mathrm{CD}_{3} \mathrm{CN}\right) \delta: 8.42$ $(\mathrm{d}, J=9.6 \mathrm{~Hz}, 1 \mathrm{H}), 7.87$ (s, 1H), 7.67 (d, $J=7.8 \mathrm{~Hz}, 1 \mathrm{H})$, $7.61 \sim 7.51(\mathrm{~m}, 1 \mathrm{H}), 7.30(\mathrm{~d}, J=9.6 \mathrm{~Hz}, 1 \mathrm{H}), 6.95(\mathrm{~d}, J=$ $2.2 \mathrm{~Hz}, 1 \mathrm{H}), 4.24(\mathrm{~s}, 2 \mathrm{H}), 3.81 \sim 3.53(\mathrm{~m}, 4 \mathrm{H}), 3.25(\mathrm{t}, J=$ $7.4 \mathrm{~Hz}, 2 \mathrm{H}), 2.53(\mathrm{~s}, 2 \mathrm{H}), 1.38(\mathrm{t}, J=7.1 \mathrm{~Hz}, 6 \mathrm{H}) ;{ }^{13} \mathrm{C}$ NMR (101 MHz, DMSO- $\left.d_{6}\right) \delta: 173.0,166.8,161.0,158.8$, $155.4,149.0$, 139.3, 131.6, 129.2, 129.0, 127.5, 126.5, $123.6,117.5,116.5,96.7,45.7,33.2,31.6,29.3,12.0$; IR (KBr) v: 3419, 2923, 2854, 1758, 1635, 1515, 1347, 1120, $1039,836 \mathrm{~cm}^{-1}$; HRMS (ESI) calcd for $\mathrm{C}_{23} \mathrm{H}_{23} \mathrm{ClNO}_{3}$ 396.1361, found 396.1353.

\section{3 染料的活化和 BSA 标记过程及染料-蛋白质共轭 物的纯化}

先将染料(1 mg)溶于 $100 \mu \mathrm{L}$ 的无水 $N, N$ 二二甲基甲 酰胺(DMF)中, 用含有 2-吗啉乙磺酸(MES) (0.1 mol• $\left.\mathrm{L}^{-1}\right) 、 N$-差欮基琥珀酰亚胺(NHS) $\left(10 \mathrm{mmol} \cdot \mathrm{L}^{-1}\right)$ 和 1-(3-二 甲氨基丙基)-3-乙基碳二亚胺(EDC) $\left(10 \mathrm{mmol} \cdot \mathrm{L}^{-1}\right.$ )的溶 液在 $37{ }^{\circ} \mathrm{C}$ 下活化 $1.5 \mathrm{~h}$. 活化后离心 $15 \mathrm{~min}$, 用 PBS 洗 涤两次得到活性酯. 将活性酯溶于制备好的含 BSA (2.5 $\mathrm{mg}$ )碳酸氢钠缓冲液 $\left(0.5 \mathrm{~mL}, 50 \mathrm{mmol} \cdot \mathrm{L}^{-1}, \mathrm{pH} 9.4\right)$ 中, 在 $25{ }^{\circ} \mathrm{C}$ 下混合液摚拌 $3 \mathrm{~h}$, 形成染料-BSA 共轭物, 再 用 Sephadex G-25 离子色谱凝胶柱分离游离染料及染料 蛋白结合物.

\section{4 染料与蛋白质比值 $(D / P)$ 的测定}

对 BSA 进行标记, 并对相应的游离染料和染料BSA 结合物进行纯化分离后测定游离染料和染料-BSA 的吸收光谱. 假设染料与 BSA 结合前后摩尔晓光系数 大致相等，则可使用下列方程计算染料蛋白比率 $(\mathrm{D} / \mathrm{P})^{[28]}$

$$
\frac{D}{P}=\frac{A_{\text {conj }(\lambda \max )} \varepsilon_{\mathrm{BSA}}}{A_{\text {conj(278) }}-x A_{\text {conj }(\lambda \max )} \varepsilon_{\text {dye }}}
$$

$\varepsilon_{\mathrm{dye}}$ 代表游离染料在最大吸收波长处的摩尔消光系数; 
$\varepsilon_{\mathrm{BSA}}=45540 \mathrm{~L} \cdot \mathrm{mol}^{-1} \cdot \mathrm{cm}^{-1}$, 代表染料-BSA 在 $278 \mathrm{~nm}$ 处 的摩尔消光系数; $x=A_{\text {dye }}(278) / A_{\text {dye }(\lambda \max )}$ 代表游离染料在 $278 \mathrm{~nm}$ 处的吸光度和在最大吸收波长处吸光度的比值; $A_{\text {conj(2max) }}$ 代表染料-BSA 复合物在最大吸收波长处的吸 光度; $A_{\mathrm{conj}(278)}$ 代表染料-BSA 复合物在 $278 \mathrm{~nm}$ 处的吸光 度.

\section{5 凝集素微阵列的制备}

将 37 种凝集素(从载体实验室 Sigma-Aldrich 和 CalBiochem 购买)分别溶解于厂家推荐的缓冲液中, 再 分别加入浓度为 $0.5 \mathrm{mg} / \mathrm{mL}$ 的 BSA 构成点样缓冲液. 用 晶芯 48 点样系统在环氧修饰后的玻片上进行点样, 再 在 $60 \%$ 湿度中孵育 $3 \mathrm{~h}$, 随后在 $37{ }^{\circ} \mathrm{C}$ 抽真空干燥 $3 \mathrm{~h}$. 干燥后先用质量分数为 $0.05 \%$ Tween -20 的 $10 \mathrm{mmol} \cdot \mathrm{L}^{-1}$ $\mathrm{PBS}, 10 \mathrm{mmol} \cdot \mathrm{L}^{-1} \mathrm{PBS}$ 各清洗玻片一次, 再用质量分数 为 $1 \% \mathrm{BSA}$ 的 $10 \mathrm{mmol} \cdot \mathrm{L}^{-1} \mathrm{PBS}$ 对芯片进行封闭 $1 \mathrm{~h}$. 后 用 $1 \times$ PBST, $1 \times$ PBS 清洗玻片 $2 \mathrm{~min}$, 离心甩干芯片. 将 纯化分离后的 $\mathbf{C y 3}$-血清或者 $\mathbf{C y 3}$-唾液和新染料-血清 或者新染料一唾液与封闭好的凝集素微阵列芯片进行杂 交, 在 $37{ }^{\circ} \mathrm{C}$ 中孵育 $3 \mathrm{~h}$ 后先用 $1 \times$ PBST 洗 2 次, 再用 $1 \times \mathrm{PBS}$ 冲洗一次, 每次冲洗 $5 \mathrm{~min}$, 离心干燥后用 Genepix 4000B 芯片扫描仪扫描芯片, 光电倍增管 (PMT) 设为 $100 \%$, 先进行预扫描, 然后选定点样区域精 确扫描. 调节明亮度和对比度达到最佳视觉效果. 用 GenePix3.0 软件从扫描结果图中获取荧光信号强度值 和背景值等信息进行分析.

\section{辅助材料(Supporting Information) 化合物 HQ1} HQ4 的 HRMS-ESI, ${ }^{1} \mathrm{H}$ NMR, ${ }^{13} \mathrm{C} \mathrm{NMR}$, IR 谱图以及其 它相关图. 这些材料可以免费从本刊网站(http://siocjournal. $\mathrm{cn} /$ )上下载.

\section{References}

[1] Larsen, M. R.; Højrup, P.; Roepstorff, P. Mol. Cell. Proteomics 2005, 4, 107.

[2] Baker, J. L.; Çelik, E.; Delisa, M. P. Trends Biotechnol. 2013, 31, 313.

[3] Zhang, L.; Luo, S.; Zhang, B. mAbs 2016, 8, 524.

[4] Fry, S. A.; Afrough, B.; Lomaxbrowne, H. J.; Lomaxbrowne, H. J.; Timms, J. F.; Velentzis, L. S.; Leathem, A. J. C. Glycobiology 2011,
$21,1060$.

[5] Smit, C. H.; Diepen, A. V.; Nguyen, D. L.; Wuhrer, M.; Hoffmann, K. F.; Deelder, A. M.; Hokke, C. H. Mol. Cell. Proteomics 2015, 14,1750 .

[6] Meany, D. L.; Hackler, L.; Zhang, H.; Chan, D. W. J. Proteome Res. 2011, 10, 1425.

[7] Lee, J. H.; Jeong, Y. R.; Kim, Y. G.; Lee, G. M. Biotechnol. Bioeng. 2017, 1721.

[8] Olkhov, R. V.; Weissenborn, M. J.; Flitsch, S. L.; Shaw, A. M. Anal. Chem. 2013, 86, 621.

[9] Kuno, A.; Uchiyama, N.; Kosekikuno, S.; Ebe, Y.; Takashima, S.; Yamada, M.; Hirabayashi, J. Nat. Methods 2005, 2, 851.

[10] Yamashita, K.; Kuno, A.; Matsuda, A.; Ikehata, Y.; Katada, N.; Hirabayashi, J.; Narimatsu, H.; Watanabe, M. Gastric Cancer 2016, $19,531$.

[11] Uchiyama, N.; Kuno, A.; Tateno, H.; Kubo, Y.; Mizuno, M.; Noguchi, M.; Hirabayashi, J. Proteomics 2008, 8, 3042.

[12] Goncalves, M. S. T. ChemInform 2009, 40, 190.

[13] Li, B.; Meng, W.-F.; Yang, M.-P.; Su, N.; Liu, X.-J.; Yang, B.-Q. Chin. J. Org. Chem. 2015, 35, 2629 (in Chinese).

(李波, 孟文斐, 杨美盼, 苏娜, 刘晓静, 杨秉勤, 有机化学, 2015, 35, 2629.)

[14] Boyarskiy, V. P.; Belov, V. N.; Medda, R.; Hein, B.; Bossi, M.; Hell, S. W. Chem.-Eur. J. 2008, 14, 1784.

[15] Zhou, J.; Yang, M.-P.; Meng, W.-F.; Cheng, Z.; Yang, B.-Q. Chin. J. Org. Chem. 2014, 34, 1646 (in Chinese).

(周佳，杨美盼，孟文斐，成昭，杨秉勤，有机化学， 2014，34， 1646.)

[16] Raghavachari, R. Carbohydr. Polym. 2000, 52, 87.

[17] Meng, W.-F.; Yang, M.-P.; Cheng, Z.; Li, S.-N.; Yang, B.-Q. Chin. J. Org. Chem. 2014, 34, 398 (in Chinese).

(孟文斐, 杨美盼, 成昭, 李少妮, 杨秉勤, 有机化学, 2014, 34, 398.)

[18] Tang, K.; Zhang, P.; Wang, S.; Qiu, N.; Zhang, F. Chin. J. Org. Chem. 2016, 36, 1700 (in Chinese).

(汤昆, 张鹏超, 王升, 邱娜, 张付利, 有机化学, 2016, 36, 1700.)

[19] Tolosa, L.; Nowaczyk, K.; Lakowicz, J. SpringerPlus 2002, 139.

[20] Markova, L. I.; Terpetschnig, E. A.; Patsenker, L. D. Dyes. Pigm. 2013, 99, 561.

[21] Hosseini, M. S.; Hashemimoghaddam, H. Talanta 2005, 67, 555.

[22] Chen, X.; Peng, X.; Cui, A.; Wang, B.; Wang, L.; Zhang, R. J. Photochem. Photobiol., A 2006, 181, 79.

[23] Wang, L. Q.; Peng, X. J.; Zhang, R.; Cui, J. N.; Xu, G Q.; Wang, F. Dyes Pigm. 2002, 54, 107.

[24] Seng, B. F.; Cheng, Z. N.; Liu, M. P. Recombinant Antibody, Science Press, Beijing 2005, pp. $440 \sim 441$ (in Chinese) (沈倍奋，陈志南，刘民培，重组抗体，科学出版社，北京，2005, pp. $440 \sim 441$.)

[25] Ge, J. F.; Arai, C.; Ihara, M. Dyes Pigm. 2008, 79, 33.

[26] Richter, C.; Ernsting, N P.; Mahrwald, R. ChemInform 2016, 47, 1217.

[27] Liu, J.; Sun, Y. Q.; Wang, P.; Zhang, J.; Guo, W. Analyst 2013, 138, 2654.

[28] Mujumdar, S. R.; Mujumdar, R. B.; Grant, C. M.; Waggoner, A. S. Bioconjugate Chem. 1996, 7, 35. 